\title{
Familles knesériennes et le problème anx limites pour l'équation difóérentielle ordinaire du second ordre
}

\author{
Par
}

\author{
Masuo HuKuHARA
}

Concernant le problème aux limites pour l'équation différentielle ordinaire du second ordre

$$
d^{2} x / d t^{2}=f(t, x, d x / d t)
$$

on sait de beaux résultats de M. Nagumo $[4,5,7]$ et de H. Okamura [1]. J'ai traité le système de deux équations différentielles

$$
d x / d t=f(t, x, y), \quad d y / d t=g(t, x, y)
$$

avec la condition aux limites

$$
x(\alpha)=\beta, \quad y\left(\alpha^{\prime}\right)=\beta^{\prime},
$$

où $\alpha, \alpha^{\prime}, \beta, \beta^{\prime}$ sont des valeurs données [6]. Comme nous avons remarqué plusieurs fois, l'équation différentielle (1) avec une condition aux limites peut se transformer au système différentiel (2) avec la condition (3).

Supposons que les fonctions $f$ et $g$ soient définies dans un compact D limité par deux plans $t=\alpha, t=\alpha^{\prime}$ et que les ensembles

$$
\mathfrak{F}=\{(\alpha, \beta, y) \in \mathfrak{D}\}, \quad \mathfrak{F}^{\prime}=\left\{\left(\alpha^{\prime}, x, \beta^{\prime}\right) \in \mathfrak{D}\right\}
$$

soient des segments de droites. Alors le problème s'énonce: Trouver une courbe solution de (2) qui joint $\stackrel{\xi}{a} \dot{a}^{\prime}$.

Donnons au lieu de (3) deux relations

$$
\varphi(x, y)=0, \quad \psi(x, y)=0
$$

et proposons-nous de trouver des solutions satisfaisant à ces relations 
respectivement pour $t=\alpha, t=\alpha^{\prime}$. On prend alors sur les plans $t=\alpha, \alpha^{\prime}$ les deux courbes $\mathfrak{F}$, $\mathfrak{F}^{\prime}$ sur lesquelles les relations (5) sont satisfaites et le problème s'énonce comme tout à l'heure.

\section{Familles de caractéristiques.}

Soient $f$ et $g$ des fonctions définies respectivement dans $\mathfrak{F}$ et $\mathfrak{F}^{\prime}$. Si $\mathfrak{F}$ est une partie de $\mathfrak{F}^{\prime}$ et si $f$ et $g$ coïncident dans $\mathfrak{F}$, nous dirons que $f$ est une partie de $g$ et que $g$ est une extension de $f$. Dans la suite nous supposerons toujours que le domaine de définition est un intervalle compact.

$\boldsymbol{F}$ étant un ensemble dont les éléments sont des fonctions, un élément $f$ de $F$ sera dit maximal s'il n'y a dans $F$ aucune extension de $f$ autre qu'elle même.

Définition 1.1. Famille de caractéristiques est l'ensemble de fonctions continues à valeurs dans $R^{n}$ (ou courbes dans $R \times R^{n}$ ) satisfaisant aux conditions suivantes et ses éléments sont appelés caractèristiques:

$1^{\circ}$ Chaque caractéristique est une fonction continue à valeurs dans $R^{n}$ définie dans un intervalle compact (qui peut se réduire à un point);

$2^{\circ}$ Une partie d'une caractéristique est aussi une caractéristique, c'est-à-dire si une fonction coinncide avec une des caractéristiques dans l'intervalle où elle est définie, elle est une caractéristique;

$3^{\circ}$ La famille est un ensemble compact dans l'espace distancié $\boldsymbol{D}=\mathbb{D}\left(R^{n+1}\right)$, où la distance $\operatorname{Dist}(\mathfrak{A}, \mathfrak{B})$ est définie par

$$
\operatorname{Dist}(\mathfrak{A}, \mathfrak{B})=\inf \left\{\delta ; O_{\S}(\mathfrak{A}) \supset \mathfrak{B}, O_{\delta}(\mathfrak{B}) \supset \mathfrak{A}\right),
$$

$O_{\delta}(\mathfrak{5})$ désignant le $\delta$-voisinage de $\mathfrak{5}$ :

$$
O_{\delta}(\tilde{\xi})=\{P ; \operatorname{dist}(P,(\xi)<\delta\} .
$$

$4^{\circ} \mathrm{Si}$ deux caractéristiques coïncident en une valeur $\alpha$ de la variable indépendante $t$, la fonction qui coïncide avec l'une d'elles pour $t \leqq \alpha$ et avec l'autre pour $t \geqq \alpha$ est aussi une caractéristique;

$5^{\circ} \quad$ Les extrémités des caractéristiques maximales se trouvent sur la frontière de l'ensemble engendré par les caractéristiques. 
Définition 1.2. Si $\boldsymbol{F}$ est une famille de caractéristiques, l'ensemble (dans $R \times R^{n}$ ) rempli par les caractéristiques de $\boldsymbol{F}$ est appelé domaine fondamental de $\boldsymbol{F}$; il est désigné par $\mathfrak{D}=\mathfrak{D}(\boldsymbol{F})$.

On a immédiatement la

Proposition 1.1. Le domaine fondamental d'une famille de caractéristiques est un ensemble compact dans $R \times R^{n}$.

Définition 1. 3. Soit $\mathfrak{D}^{\prime}$ une partie fermée de $\mathfrak{D}(\boldsymbol{F})$. L'ensemble des caractéristiques de $\boldsymbol{F}$ contenues dans $\mathfrak{D}^{\prime}$ est appelée sous-famille de $\mathbb{F}$ restreinte à $\mathfrak{D}^{\prime}$. Nous la désignons par $\boldsymbol{F}\left(\mathfrak{D}^{\prime}\right)$.

On voit sans peine que l'on a les propositions suivantes.

Proposition 1.2. Si $\mathfrak{D}^{\prime}$ est une partie fermée de $\mathfrak{D}$, la sousfamille de $\boldsymbol{F}$ restreinte à $\mathfrak{D}^{\prime}$ est aussi une famille de caractéristiques.

Proposition 1.3. Soient $\left\{\mathfrak{F}_{\lambda} ; \lambda \in \Lambda\right\}$ un ensemble de parties compactes de $\mathfrak{D}$. Si leur intersection $\mathfrak{F}=\cap \mathfrak{F}_{\lambda}$ n'est pas vide, on a

$$
\boldsymbol{F}(\mathfrak{s})=\bigcap \mathbb{F}\left(\mathfrak{G}_{\lambda}\right)
$$

c'est-à-dire la sous-famille de $\boldsymbol{F}$ restreinte à $\mathfrak{F}$ est l'intersection des sous-familles de $\mathbf{F}$ restreintes aux $\mathfrak{F}_{\lambda}$.

Dèfinition 1.4. (5 étant une partie quelconque de $R \times R^{n}$, l'ensem-

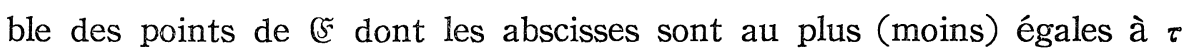
est appelé ensemble (r tronqué à droite (gauche) par l'hyperplan $t=\tau$. Nous le désignons par $\mathfrak{r}_{\tau}^{d}\left(\xi_{\tau}^{g}\right)$.

Définition 1.5. $\mathbb{F}$ étant une famille de caractéristiques, $\boldsymbol{F}\left(\mathfrak{S}_{\tau}^{d}\right)$ est appelé sous-famille de $\mathbb{F}$ tronquée à droite par l'hyperplan $t=\tau$, et nous le désignons par $\mathbb{F}_{\tau}^{d}$. Nous définissons de même sous-famille tronquée à gauche; elle est désignée par $\mathbb{F}_{\tau}^{g}$.

\section{Classification des points frontières.}

Définition 2.1. Les extrémités gauches des caractéristiques maximales sont appelés points extrêmes gauches de $\mathfrak{D}$ et l'ensemble des 
points extrêmes gauches frontière gauche de $\mathfrak{D}$; la frontière gauche est désignée par $\mathfrak{B}^{E}=\mathfrak{B}^{g}(F)$. On définit de même points extrêmes droits et frontière droite; celle-ci est désignée par $\mathfrak{B}^{d}=\mathfrak{B}^{d}(\mathbb{F})$.

Définition 2.2. Une caractéristique $x$ est applée demi-caractéristique gauche maximale si son extrémité gauche appartient à $\mathfrak{S}^{g}$. On définit de même demi-caractéristique droite maximale.

Nous désignons par $\mathbb{F}^{+}(\mathfrak{F})$ l'ensemble des parties des demi-caractéristiques droites maximales dont les extrémités gauches se trouvent dans (5. La famille $\boldsymbol{F}^{+}(\sqrt{5})$ tronquée à droite par l'hyperplan $t=\tau$ est désignée par $\mathbb{F}_{\tau}^{+}(\mathbb{F})$. On définit de même l'ensemble des caractéristiques $\boldsymbol{F}^{-}(\mathfrak{F})$ et $\boldsymbol{F}_{\tau}^{-}(\mathfrak{F})$.

On a immédiatement la

Proposition 2.1. Si (5 est une partie compacte de $\mathfrak{D}, \mathbb{F}^{ \pm}(\mathbb{F})$ et $\boldsymbol{F}_{\tau}^{ \pm}([)$sont des familles de caractéristiques.

Remarque. La réunion $\boldsymbol{F}^{+}(\mathfrak{F}) \cup \boldsymbol{F}^{-}(\mathfrak{F})$ n'est pas une famille de caractéristiques.

Définition 2.3. Considérons un point frontière $A$ qui n'est pas un point extrême droit. Chaque caractéristique maximale de $\mathbb{F}^{+}(A)$ est définie dans un intervalle avec une longueur positive. On peut distinguer deux cas suivant que les points de $3^{+}(A)=\mathscr{D}\left(\boldsymbol{F}^{+}(\mathfrak{F})\right)$ assez voisins de $A$ se trouvent à l'intérieur de $\mathfrak{D}$ sauf le point $A$ ou non. Dans le premier cas $A$ est un point isolé de $\mathfrak{B} \cap \mathfrak{Z}^{+}(A)$ tandis que dans le second cas $A$ en est un point d'accumulation. Désignons par $\mathfrak{B}^{+}$ l'ensemble des points frontières tels que l'on ait le premier cas, et par $\mathfrak{B}_{+}$celui des points frontières tels que l'on ait le second cas. On définit de même les ensemble $\mathfrak{B}^{-}, \mathfrak{B}_{-}$.

\section{Zone d'émission.}

Définition 3.1. Le domaine fondamental $\mathfrak{D}\left(\boldsymbol{F}^{+}(\mathfrak{F})\right)$ de la famille $F^{+}(\mathfrak{F})$ est applé zone d'émission à droite de $\sqrt{5}$; il est désigné par $3^{+}(\mathfrak{F})=3^{+}(\mathbb{F} ; \boldsymbol{F})$. L'ensemble $3^{+}(\mathfrak{F} ; \boldsymbol{F})$ tronqué à droite par l'hyperplan $t=\tau$ est désigné par $Z_{\tau}^{+}(\xi)=Z_{\tau}^{+}(\xi ; F)$. On définit de même 
zone d'émission à gauche de $\widetilde{s}$ et la zone d'émission à gauche de (s tronquée par l'hyperplan $t=\tau$ et on les désigne par $\widehat{S}^{-}(\widetilde{S})=\mathfrak{S}^{-}(\tilde{S} ; \mathbb{F})$ et $3 \bar{\tau}(\widetilde{F})=3 \bar{\tau}(\widetilde{F} ; \mathbb{F})$. La réunion $3(\mathbb{F})=3^{+}(\sqrt{5}) \cup 3^{-}(\sqrt{5})$ est applée zone d'émission de

Il est clair que l'on a les propositions suivantes.

Proposition 3. 1. La sous-famille de $F$ restreinte à $3^{+}((\overrightarrow{5})$ coïncide avec $\mathbb{F}^{+}$(5).

Remarque. II est clair que si l'on a une proposition relative à la famille $\mathbb{F}^{+}(\xi)$, on a une proposition analogue relative à la famille $\mathbb{F}^{-(}(5)$. Par exemple, l'analogue de la proposition 3.1 s'énonce comme il suit:

La sous-famille de $\mathbb{F}$ restreinte à $3^{-}$(अ) coincide avec $\mathbb{F}^{-(}$(अ) .

Dorénavant, nous n'énoncerons pas, pour être court, l'analogue d'une proposition, si on l'obtient par simple intervertissement du sens.

Proposition 3.2. Si $\tilde{\xi}_{1}$ et $\underline{\mathfrak{G}}_{2}$ sont deux parties compactes de (D), on $a$

$$
\boldsymbol{F}^{+}\left(\mathfrak{F}_{1} \cup \mathfrak{F}_{2}\right)=\boldsymbol{F}^{+}\left(\mathfrak{F}_{1}\right) \cup \boldsymbol{F}^{+}\left(\mathfrak{F}_{2}\right) .
$$

Proposition 3. 3. Si $\varsigma^{\prime}$ est une partie compacte de $Z^{+}(\xi)$, on a

$$
\boldsymbol{F}^{+}\left(\varsigma^{\prime}\right)=\left(\boldsymbol{F}^{+}(\xi)\right)^{+}\left(\xi^{\prime}\right) \text {. }
$$

\section{Familles knesériennes.}

Définition 4.1. Un point $A \in \mathfrak{D}$ d'abscisse $\alpha$ est appelé point knesérien à droite dans les cas suivants:

$1^{\circ} A \in \mathfrak{B}^{d}$;

$2^{\circ} \quad A$ appartient à $\mathfrak{B}^{+}$ou à l'intérieur de $\mathfrak{D}$ et la section de $3^{+}(A)$ par l'hyperplan $t=\tau$ est un continu pourvu que la différence $\tau-\alpha$ est positive et assez petite;

$3^{\circ} A \in \mathfrak{B}_{+}$et la réunion de $3_{\tau}^{+}(A) \cap \mathfrak{R}$ et de la section de $3^{+}(A)$ par l'hyperplan $t=\tau$ est un continu pourvu que la différence $\tau-\alpha$ soit positive et assez petite.

On définit de même point knesérien à gauche. 
Définition 4.2. Si tout point de (D) est kntsérien à droite et si de plus $\mathfrak{B}^{+}$est une partie ouverte de $\mathfrak{B}$ et est contenue dans $\mathfrak{B}^{g}, \boldsymbol{F}$ est appelée famille knesérienne à droite. On définit de même famille knesérienne à gauche. Une famille knesérienne à droite et à gauche est appelée famille knesérienne.

Proposition 4.1. Si $\mathbb{F}$ est une famille knesérienne à droite, les familles $\boldsymbol{F}_{\tau}^{d}, \boldsymbol{F}_{\tau}^{g}$ le sont aussi.

$1^{\circ} \quad F_{\tau}^{g}$ est knesérienne à droite, $\operatorname{car}\left(\boldsymbol{F}_{\tau}^{g}\right)^{+}(P)$ coïncide avec $\boldsymbol{F}^{+}(P)$ pour $P \in \mathfrak{D}_{\tau}^{g}$.

$2^{\circ} \quad \mathbb{F}_{\tau}^{d}$ est knesérienne à droite, car $\left(\boldsymbol{F}_{\tau}^{d}\right)_{\tau}^{+}(P)$ coïncide avec $\boldsymbol{F}_{\tau}^{+}(P)$ sauf pour les points qui se trouvent sur l'hyperplan $t=\tau$ et ceux-ci appartiennent à $\mathfrak{B}^{d}\left(\boldsymbol{F}_{\tau}^{d}\right)$.

Proposition 4.2. Soit $\boldsymbol{F}$ une famille knesérienne à droite. Si (5 est une partie continue de $\mathfrak{D}$, l'intersection

$$
\mathfrak{E}=\mathfrak{Z}^{+}(\mathfrak{F}) \cap\left(\mathfrak{B}_{+} \cup \mathfrak{B}^{d}\right)
$$

est un continu.

En effet, $\sqrt{5}$ est un compact. Supposons que $\sqrt{5}$ soit une réunion de ses deux parties fermées $\mathfrak{F}_{1}$ et $\mathfrak{F}_{2}$. Les ensembles

$$
3_{i}=\left\{P \in 3^{+}(\Im) ; 3^{+}(P) \cap \bigodot_{i} \neq \phi\right\}, \quad i=1,2,
$$

sont des parties fermées de $3^{+}(\mathfrak{F})$ et leur réunion coïncide avec $3^{+}(\mathfrak{F})$. Celui-ci est un continu parce que tout son point est joint à $\mathfrak{5}$ par une caractéristique. Par suite l'intersection $\mathcal{B}_{1} \cap \mathbb{Z}_{2}$ n'est pas vide. Soit $A$ un de ses points ayant la plus grande abscisse $\alpha$.

Si $A$ n'appartenait pas à $\mathfrak{B}^{d} \cup \mathfrak{B}_{+}$, la section $\subseteq$ de $\mathfrak{Z}^{+}(A)$ par l'hyperplan $t=\tau$ serait un continu non vide pourvu que la différence $\tau-\alpha$ soit positive et assez petite. $\widetilde{S}$ serait alors la réunion de ses deux parties fermées

$$
\mathfrak{S}_{i}=\left\{P \in \mathfrak{S} ; \mathcal{Z}^{+}(P) \cap \mathfrak{S}_{i} \neq \phi\right\}, \quad i=1,2 .
$$

L'intersection $\mathfrak{\subseteq}_{1} \cap \mathfrak{S}_{2}$, qui est ure partie de $\mathfrak{Z}_{1} \cap \mathfrak{Z}_{2}$, contiendrait donc au moins un point ayant une abscisse plus grande que $\alpha$ contrairement à la définition de $\alpha$. 
$A$ appartient donc à $\mathfrak{B}^{d} \cup \mathfrak{B}_{+}$. Si $A \in \mathfrak{B}^{d}, A$ appartient évidemment à $\mathfrak{\mho}_{1} \cap \mathfrak{夭}_{2}$. Considérons dorc le cas de $A \in \mathfrak{V}_{+}$.

ऽ ayant la même signification que plus haut, l'ensemble

$$
\mathfrak{A}=\left(3_{\tau}^{+}(A) \cap \mathfrak{B}\right) \cup \mathfrak{S}
$$

est un continu pourvu que la différence $\tau-\alpha$ soit positive et assez petite.

$$
\mathfrak{N}_{i}=\left\{P \in \mathfrak{A} ; \mathfrak{Z}^{+}(P) \cap \mathfrak{S}_{i} \neq \phi\right\}, \quad i=1,2,
$$

sont des parties fermées de $\mathfrak{A}$ et leur réunion coïncide avec $\mathfrak{A}$. Donc l'intersection $\mathfrak{U}_{1} \cap \mathfrak{U}_{2}$ n'est pas vide. Mais elle ne peut contenir aucun point d'abscisse plus grande que $\alpha$. Donc elle ne contient que le point A.

Si $A$ n'appartenait pas à $\mathbb{S}_{1}$, on pourrait supposer, en prerant $\tau-\alpha$ assez petite, que $Z_{\tau}^{+}(A)$ ne contienne aucun point de $\mathfrak{F}_{1}$. On a alors

$$
3_{\tau}^{+}(A) \cap \mathfrak{B} \subset \mathfrak{V}_{2} .
$$

$A$ appartenant à $\mathfrak{U}_{1}, \mathfrak{S}$ contiendrait des points de $\mathfrak{U}_{1}$. Alors $\mathfrak{U}_{1}^{\prime}=\mathfrak{U}_{1} \cap \mathfrak{S}$ et $\mathfrak{U}_{2}$ seraient des parties fermées non vides de $\mathfrak{A}$ et leur réunion coïnciderait avec $\mathfrak{A}$. Donc $\mathfrak{U}_{1}^{\prime} \cap \mathfrak{A}_{2}$ ne serait pas vide et $\mathfrak{U}_{1} \cap \mathfrak{A}_{2}$ contiendrait des points autres que $A$. C'est absurde.

$A$ appartient donc à l'intersection $\mathfrak{夭}_{1} \cap \mathfrak{F}_{2}$. On en conclut que $\sqrt{5}$ est un continu.

\section{Familles $\hat{\boldsymbol{F}}^{+}(\sqrt{5})$.}

Définition 5.1. Soit $\mathbb{F}$ une famille de caractéristiques. L'ensemble des caractéristiques de $\mathbb{F}^{-}(\mathfrak{F})$ qui joignent $\mathfrak{F}$ à $\mathfrak{B}^{d} \cup \mathfrak{B}_{+}$se désigne par $\widehat{\boldsymbol{F}}^{+}(\mathbb{F})$. La notation $\widehat{\boldsymbol{F}}^{-}(\mathfrak{F})$ a la signification analogue.

Proposition 5.1. $\widehat{F}^{+}(P)$ est un ensemble fermé qui depend de $P$ d'une manière semi-continue supérieurement.

Considérons en effet une suite $\left\{P_{k}\right\}$ extraite de $\mathfrak{D}$ et convergeant vers $P$ et supposons qu'une suite $\left\{r_{, k}\right\}$ telle que $x_{k} \in \widehat{F}^{+}\left(P_{k}\right)$ converge vers $x$. Il suffit de montrer que $r$. appartient à $\widehat{F^{+}}(P)$.

L'extrémité droite $Q_{k}$ de $x_{k}$ appartient à $\mathfrak{B}^{d} \cup \mathfrak{B}_{+}$et la suite $\left\{Q_{k}\right\}$ 
converge vers un point $Q$ qui appartient nécessairement à $\mathfrak{s}^{d} \cup \mathfrak{B}_{T}$. Les extrémités de $\mathfrak{r}$ sont évidemment $P$ et $Q$. $\underset{c}{ }$ appartient donc à $\widehat{F}^{+}(P)$. La de nonstration montre aussitôt que $\widehat{F}^{+}(P)$ est un ensemble fermé. Car si l'on prend $P_{k}=P,\left\{x_{k}\right\}$ est une suite extraite de $\widehat{F}^{+}(P)$ et alors sa limite $x$ appartient aussi à $\widehat{F}^{+}(P)$.

Proposition 5.2. Si $\mathbb{F}$ est une famille knesérienne à droite, $\widehat{F}^{+}(A)$ est un continu quel que soit $A \in \mathfrak{D}$.

Il suffit de montrer que $\widehat{\mathbb{F}}^{+}(A)$ est bien enchaîné. Pour cela, nous prenons deux éléments quelconques z̧; et $\mathfrak{\eta}$ de $\widehat{F}^{+}(A)$ et nous montrerons que l'on peut les joindre par une chaîne d'éléments de $\widehat{F}^{+}(A)$ à chaînors inférieurs à un nombre positif arbitraire $\varepsilon$.

D'après la compacité de $\mathbb{F}$, on peut faire correspondre à $\rho>0$ un nombre $f(\rho)>0$ de manière que l'on ait

$$
\left|z(t)-z\left(t^{\prime}\right)\right|<\rho
$$

pour $z \in \mathbb{F},\left|t-t^{\prime}\right|<f(\rho)$. Soit $\alpha$ l'abscisse de $A$ et $\beta$ la plus grande des abscisses des points de $3^{+}(A)$. Prenons une suite croissante $\alpha=\alpha_{0}$, $\alpha_{1}, \cdots, \alpha_{N-1}, \alpha_{N}=\beta$ telle que

$$
\alpha_{k}-\alpha_{k-1}<\min \{\varepsilon / 2, f(\varepsilon / 2)\}, \quad k=1,2, \cdots, N,
$$

et désignons par $\mathfrak{F}_{k}$ la réunion de la section $\widetilde{\subseteq}_{k}$ de $3^{+}(A)$ par l'hyperplan $t=\alpha_{k}$ et de l'ensemble

$$
3_{\alpha_{k}}^{+}(A) \cap\left(\mathfrak{B}^{d} \cup \mathfrak{B}_{+}\right) \text {. }
$$

D'après les propositions 4.1 et $4.2, \mathfrak{F}_{k}$ est un continu.

Désignons par $\mathscr{L}_{k}={\widehat{F_{\alpha}}}_{\alpha_{k}}^{+}(A)$ l'ensemble des caractéristiques de $\boldsymbol{F}(A)$ limitées par $A$ et $\mho_{k}$. Nous voulons montrer par récurrence que deux éléments quelconques de $\mathscr{F}_{k}$ peuvent se joindre par une chaîne d'éléments de $\mathscr{H}_{k}$ à chaînons inférieurs à $\varepsilon$ telle que les extrémités droites forment une chaîne à chaînons inférieurs à $\rho_{k}$; les conditions auxquelles doit satisfaire la suite $\left\{\rho_{k}\right\}$ seront données dans les lignes suivantes.

Soient $\mathfrak{x}$ et $\mathfrak{y}$ deux éléments quelconques de $\mathfrak{F}_{1}$, et $P$ et $Q$ leurs extrémités droites. $\mathbb{F}_{1}$ étant un continu, on peut les joindre par une chaîne d'éléments de $\mathfrak{F}_{1}$ à chaînons inférieurs à $\rho_{1}: P=P_{1}, P_{2}, \cdots, P_{m}=Q$. 
Prenons un élément $\mathfrak{x}_{i}$ de $\mathscr{F}_{1}$ dont l'extrémité droite est $P_{i}, \quad(i=1,2$, $\cdots, m)$. Nous posons en particulier $\mathfrak{x}_{1}=\mathfrak{x}, \mathfrak{x}_{m}=\mathfrak{l}$.

Puisque l'on a $\mathfrak{r}_{i}(\alpha)=\mathfrak{x}_{(}(\alpha)$ et $\alpha_{1}-\alpha<f(\varepsilon / 2)$, on a

$$
\left|\mathfrak{x}_{i}(t)-\mathfrak{x}(\alpha)\right|<\varepsilon / 2,
$$

d'où

$$
\operatorname{Dist}\left(\mathfrak{x}_{i}, \mathfrak{x}_{j}\right)<\varepsilon .
$$

$\mathfrak{x}_{1}, \mathfrak{x}_{2}, \cdots, \mathfrak{x}_{m}$ forment donc une chaîne à chaînons inférieurs à $\varepsilon$.

Considérons maintenant le cas de $k$ quelconque. Nous prenons deux éléments quelconques $x$, et $\mathfrak{y}$ de $\mathscr{F}_{k}$, et désignons par $\mathfrak{x}^{\prime}$ et $\mathfrak{y}^{\prime}$ les arcs partiels de $x$ et de $y$ limités par $A$ et $\mathfrak{F}_{k-1}$. Par l'hypothèse de récurrence, on peut construire une chaîne d'éléments de $\mathscr{F}_{k-1}$ à chaînons inférieurs à $\varepsilon: \mathfrak{c}^{\prime}=\mathfrak{x}_{1}^{\prime}, r_{2}^{\prime}, \cdots, \mathfrak{x}_{m}^{\prime}=\mathfrak{l}^{\prime}$ telle que leurs extrémités droites $P_{1}^{\prime}, P_{2}^{\prime}, \cdots, P_{m}^{\prime}$ forment une chaîne à chaînons inférieurs à $\rho_{k-1}$.

D'après la proposition 5.1, $\widehat{F}_{\alpha_{k}}^{+}(P)$ dépend de $P$ d'une manière semi-continue supérieurement lorsque $P$ parcourt $\mathfrak{F}_{k-1}$. Si donc on prend $\rho_{k-1}$ assez petit après avoir défini $\rho_{k}$, on aura

$$
\operatorname{dist}\left(\widehat{\mathbb{F}}_{\alpha_{k}}^{+}\left(P_{i}^{\prime}\right), \widehat{F}_{\alpha_{k}}^{+}\left(P^{\prime}{ }^{\prime}\right)\right)<\min \left\{\rho_{k} / 3, f\left(\rho_{k} / 3\right\}\right. \text {. }
$$

On peut donc choisir $\mathfrak{y}_{2}^{\prime \prime} \in \mathbb{F}_{\alpha_{k}}^{+}\left(P_{\imath}^{\prime}\right)$ et $\mathfrak{x}_{i+1}^{\prime \prime} \in \mathbb{F}_{\alpha_{k}}^{+}\left(P_{i+1}^{\prime}\right)$ de manière que lon ait

$$
\operatorname{Dist}\left(\mathfrak{y}_{\imath}^{\prime \prime}, x_{1+1}^{\prime \prime}\right)<\min \left\{\rho_{k} / 3, f\left(\rho_{k} / 3\right)\right\} \text {. }
$$

Soient $Q_{i}^{\prime \prime}, P_{i+1}^{\prime \prime}$ les extrémités droites de $\eta_{i}^{\prime \prime}, x_{i+1}^{\prime \prime}$. Nous prenons pour $\mathbb{P}_{1}^{\prime \prime}$ et $Q_{m}^{\prime \prime}$ les extrémités dioites de $x$ et $\mathfrak{l}$ et pour $\mathfrak{x}_{1}^{\prime \prime}$ et $y_{m}^{\prime \prime}$ les arcs partiels respectifs de $x$ et i) dont les extrémités sont $P_{1}^{\prime}, P_{1}^{\prime \prime}$ et $Q_{{ }^{\prime \prime}}^{\prime}, Q_{m}^{\prime \prime}$.

Si aucun des points $P_{2}^{\prime}$ et $P_{t+1}^{\prime}$ r'apcartiert à l'hyperplan $t=\alpha_{k-1}$, iis appartiennent à $\widetilde{E}_{1}$. Alors on prend pour $x_{i}^{\prime \prime}, n_{i}^{\prime \prime}$ les points $P_{2}^{\prime}, P^{\prime}{ }^{\prime}$ eux-mêmes de sorte que $Q_{2}^{\prime \prime}=P_{i}^{\prime}, P_{i+1}^{\prime \prime}=P_{i+1}^{\prime}$.

Considérons le cas où un au moins des points $Q_{\imath}^{\prime}$ et $P_{i+1}^{\prime}$ appartient à l'hyperplan $i=a_{r-1}$. Soient $\tau$ et $\tau^{\prime}$ les abscisses des poirts $P_{1+1}^{\prime \prime}, Q_{\imath}^{\prime \prime}$ et supposons par exemple que lon ait $\tau^{\prime} \leqq \tau$. Soit $\tau^{\prime \prime}$ une valeur telle que

$$
\operatorname{dist}\left(\tilde{Q}_{\imath}, P_{\imath+1}^{\prime \prime}\right)=\operatorname{dist}\left(\iota_{i}^{\prime \prime}, P_{i+1}^{\prime \prime}\right)
$$

où $\tilde{Q_{\imath}}=\left(\tau^{\prime \prime}, \eta_{\imath}^{\prime \prime}\left(\tau^{\prime \prime}\right)\right)$. On a alors 


$$
\begin{aligned}
& 0 \leqq \tau^{\prime}-\tau^{\prime \prime} \leqq \tau-\tau^{\prime \prime} \\
& \leqq \operatorname{dist}\left(\mathfrak{y}_{i}^{\prime \prime}, P_{i+1}^{\prime \prime}\right) \\
& \leqq \operatorname{Dist}\left(\mathfrak{y}_{i}^{\prime \prime}, \mathfrak{c}_{i+1}^{\prime \prime}\right) \\
& <\min \left\{\rho_{k} / 3, f\left(\rho_{k} / 3\right)\right\} .
\end{aligned}
$$

On a donc

$$
\begin{aligned}
& \operatorname{dist}\left(Q_{i}^{\prime \prime}, P_{i+1}^{\prime \prime}\right) \\
\leqq & \operatorname{dist}\left(Q_{i}^{\prime \prime}, \tilde{Q}_{i}\right)+\operatorname{dist}\left(\tilde{Q_{i}}, P_{i+1}^{\prime \prime}\right)<\rho_{k} .
\end{aligned}
$$

La réunion $\mathfrak{S}_{i k}$ de la section de $3^{+}\left(P_{i}^{\prime}\right)$ par l'hyperplan $t=\alpha_{k}$ et de l'ensemble

$$
\mathfrak{Z}_{\alpha_{k}}^{+}\left(P_{i}^{\prime}\right) \cap\left(\mathfrak{B}^{d} \cup \mathfrak{B}_{+}\right)
$$

est un continu qui contient $P_{i}^{\prime \prime}$ et $Q_{i}^{\prime \prime}$. Si donc $P_{i}^{\prime \prime} \neq Q_{i}^{\prime \prime}$, on peut les joindre par une chaîne de points de $\mathfrak{F}_{i k}$ à chaînons inférieurs à $\rho_{k}$ : $\left\{P_{i j}^{\prime \prime} ; j=1,2, \cdots, m_{i}\right\}$. Soit $\mathfrak{x}_{2 j}^{\prime \prime}$ une caractéristique de $\widehat{F}_{\alpha_{k}}^{+}\left(P_{\imath}^{\prime}\right)$ dont l'extrémité droite est $P_{i j}^{\prime \prime}$. Puisque l'un au moins de $P_{i}^{\prime \prime}$ et $Q_{i}^{\prime \prime}$ est différent de $P_{i}^{\prime}$, l'abscisse $\beta_{i}$ de $P_{i}^{\prime}$ est au moins égale à $\alpha_{k-1}-\rho_{k-1}$. L'abscisse $\beta_{t j}^{\prime \prime}$ de $P_{i j}^{\prime \prime}$ est au plus égale à $\alpha_{k}$. Si donc $\rho_{k-1}$ est assez petit de sorte que

$$
\alpha_{k}-\alpha_{k-1}+\rho_{k-1}<\min \{\varepsilon / 2, f(\varepsilon / 2)\},
$$

on a

$$
\operatorname{dist}\left(\mathfrak{d}_{i}^{\prime}\left(\beta_{i}\right), \mathfrak{d}_{i j}^{\prime \prime}(t)\right)<\varepsilon / 2
$$

pour $\beta_{i} \leqq t \leqq \beta_{i j}^{\prime \prime}$. On en conclut que l'on a

$$
\operatorname{Dist}\left(\mathfrak{G}_{i j}^{\prime \prime},{\underset{r}{r}, j+1}^{\prime \prime}\right)<\varepsilon \text {. }
$$

En joignant $\mathfrak{x}_{i}^{\prime}$ à $\mathfrak{x}_{i j}^{\prime \prime}$ bout à bout, on obtient une caractéristique $x_{i j}$ de $\widehat{F}_{\alpha_{k}}^{+}(A)$ et les caractéristiques $\left\{x_{i j} ; j=1, \cdots, m_{i} ; i=1, \cdots, m\right\}$ forment une chaîne à chaînons inférieurs à $\varepsilon$ joignant $\mathfrak{c}$ et $\mathfrak{y}$ et les extrémités droites forment une chaîne à chaînons inférieurs à $\rho_{k}$ joignant les extrémités droites de $\mathfrak{x}$ et $\mathfrak{y}$.

Nous voulons maintenant établir une proposition réciproque dont l'énoncé voici.

Proposition 5.3. Soit $\mathbb{F}$ une famille de caractéristiques satisfaisant aux conditions suivantes: 
1) $\widehat{\boldsymbol{F}}^{+}(P)$ est un continu pour tout $P \in \mathfrak{D}$;

2) $\mathfrak{B}^{+}$est une partie ouverte de $\mathfrak{B}$ telle que $\mathfrak{B}^{+} \subset \mathfrak{B}^{g}$;

Alors $\boldsymbol{F}$ est une famille knesérienne à droite.

Considérons le cas de $A \notin \mathfrak{B}^{d} \cup \mathfrak{B}_{+}$. Toute caractéristique de $\widehat{\boldsymbol{F}}^{+}(A)$ existe dans un intervalle assez petit $[\alpha, \tau]$, où $\alpha$ désigne l'abscisse du point $A$.

Si la section $\mathfrak{S}$ de $3^{+}(A)$ par l'hyperplan $t=\tau$ est une réunion de ses deux parties fermées $\widetilde{\Im}_{1}, \mathfrak{S}_{2}, \widehat{F}^{+}(A)$ est la réunion de ses deux parties fermées

$$
\widehat{\boldsymbol{F}}^{+}(A) \cap \mathbb{F}\left(\mathfrak{S}_{i}\right), \quad i=1,2,
$$

et, puisque $\widehat{F}^{+}(A)$ est un continu par hypothèse,

$$
\widehat{\mathbb{F}}^{+}(A) \cap \boldsymbol{F}\left(\widetilde{\Im}_{1}\right) \cap \mathbb{F}\left(\widetilde{\Xi}_{2}\right)
$$

n'est pas vide. On en conclut que $\mathfrak{\subseteq}_{1} \cap \mathfrak{\Im}_{2}$ n'est pas vide. Par suite $\mathfrak{\subseteq}$ est un continu.

On peut démontrer de même que si $A \in \mathfrak{ß}_{+}^{2}$

$$
\mathfrak{S}=\mathfrak{S} \cup\left(\mathfrak{Z}_{\tau}^{+}(A) \cap\left(\mathfrak{B}_{+} \cup \mathfrak{B}^{d}\right)\right)
$$

est un continu pourvu que $\tau-\alpha$ soit positive et assez petite, $\alpha$ désignant l'abscisse du point $A$. Si l'on fait correspondre à une caractéristique de $\widehat{\vec{F}}^{+}(A)$ son extrémité, on obtient une application continue. Par suite un point quelconque de $\mathfrak{B}_{+}$est aussi knesérien à droite.

\section{Familles proprement knesériennes.}

Définition 6.1. Soit $\mathbb{F}$ une famille de caractéristiques. Nous désignons par $\widetilde{\boldsymbol{F}}$ l'ensemble des caractéristiáues maximales de $\mathbb{F}$. Si $P$ appartient à $\mathfrak{S}, \widetilde{F}^{+}(P)$ représente l'ensemble des caractéristiques maximales de $\boldsymbol{F}^{+}(P)$. (ㄷ étant une partie compacte de $\mathfrak{S}$, nous posons

$$
\widetilde{F}^{+}(\mathfrak{s})=\bigcup\left\{\widetilde{\boldsymbol{F}}^{+}(P) ; P \in(\mathfrak{s}\}\right. \text {. }
$$

Nous définissons de même $\widetilde{\boldsymbol{F}}^{-}(\mathfrak{\xi})$. Nous désignons en particuliers par $\widetilde{\boldsymbol{F}}^{ \pm}$les ensembles $\widetilde{\boldsymbol{F}}^{ \pm}(\mathfrak{D})$. 
Proposition 6.1. Les trois conditions suivantes sont équivalentes:

(i) $\widetilde{\boldsymbol{F}}^{+}$est un compact;

(ii) $\widetilde{F}^{+}(P)$ dépend de $P$ d'une manière semi-continue supérieurement;

(iii) $\mathfrak{B}^{d}$ est un compact.

(i) $\Rightarrow$ (ii). Considérons une suite $\left\{P_{k}\right\}$ extraite de $\mathfrak{D}$ et convergeant vers $P$ et une suite $\left\{x_{k}\right\}$ convergeant vers $x$ et telle que $\mathfrak{c}_{k} \in \widetilde{F}^{+}\left(P_{k}\right)$. Il suffit de montrer que $\mathfrak{x}$ appartient à $\widetilde{\mathbb{F}}^{+}(P)$.

D'après la compacité de $\widetilde{F}^{+}$, l'extrémité droite de $r$ est un point extrême droit de $\mathfrak{D}$. L'extrémité gauche de $x$ est évidemment $P$. appartient donc à $\widetilde{F}^{+}(P)$.

(ii) $\Rightarrow$ (iii). Considérons une suite $\left\{B_{k}\right\}$ extraite de $\mathfrak{B}^{d}$ et convergeant vers $B$. Il suffit de montrer que $B$ est un point extrême droit de $\mathfrak{D}$.

Il existe une caractéristique $\Upsilon_{k}$ dont l'extrémité droite est $B_{k}$. Soit $A_{k}$ l'extrémité gauche de $r_{k} . r_{k}$ appartient à $\widetilde{F}^{+}\left(A_{k}\right)$. En prenant, s'il est nécessaire, une suite partielle, nous pouvons supposer que la suite $\left\{\mathfrak{x}_{k}\right\}$ converge vers une caractéristique $\mathfrak{c}_{0}$ Alors les suites $\left\{A_{k}\right\}$. $\left\{B_{k}\right\}$ convergent respectivement vers l'extrémité gauche $A$ et l'extrémité droite $B$ de $x$ et la semi-continuité supérieure de $\widetilde{F}^{+}(P)$ implique que x. appartient à $\widetilde{\mathbb{F}^{+}}(A)$. $B$ est donc un point extrême droit.

(iii) $\Rightarrow$ (i). $\quad \widetilde{\mathbb{F}}^{+}$étant une partie d'un ersemble compact $\mathbb{F}$, il suffit de montrer que $\widetilde{F}^{+}$est fermée. Par suite il suffit de montrer que si une suite $\left\{r_{k}\right\}$ extraite de $\widetilde{\mathbb{F}^{+}}$converge vers $x, x$ appartient à $\widetilde{\mathbb{F}}^{+}$.

Si $B_{k}$ est l'extrémité droite de $\hat{\imath}_{k},\left\{B_{i}\right\}$ converge vers l'extrémité droite $B$ de r. $r_{k}$ apparterant à $\widetilde{F}^{+}, B_{k}$ est un poirt extrême droit, et I'hypothèse (iii) implicue que $B$ est aussi un point extrême droit. $\Re$ appaitient dorc à $\widetilde{F}^{+}$.

Définition 6.2. Un point $A$ de $D$ d'abscisse $\alpha$ est appelé point proprement knesérien à droite si l'une des conditions suivantes est remplie: 
$1^{\supset} \quad A \in \mathfrak{B}^{d}$;

$2^{\circ}$ Chaque caractéristique $\mathbb{F}^{+}(A)$ perce l'hyperplan $t=\tau$ et la section de $3^{+}(A)$ par lui est un continu pour $\tau-\alpha>0$ assez petite.

On définit de même point proprement knesérien à gauche.

Remarque. Un point knesérien à droite est un point proprement knesérien à droite et réciproquement, si le point appartient à $\mathfrak{B}^{a} \cup \mathfrak{B}^{+}$ ou à l'intérieur de $\mathfrak{D}$. On n'a plus l'équivalence des deux conditions si le point appartient à $\mathfrak{B}_{+}$.

Définition 6. 3. Une famille de caractéristiques est appelée famille proprement knesérienne à droite si tout point de $\mathfrak{D}$ est point proprement knesérien à droite. On définit de même famille proprement knesérienne à gauche. Si $\mathbb{F}$ est à la fois famille proprement knesérienne à droite et à gauche, elle est appelée famille proprement knesérienne.

Proposition 6.2. Si $\mathbb{F}$ est une famille proprement knesérienne $\grave{a}$ droite $\mathfrak{Z}^{+}(A) \cap \mathfrak{B}^{d}$ est un continu pour chaque $A \in \mathfrak{D}$.

Supposons le contraire. $3^{+}(A) \cap \mathfrak{B}^{d}$ est alors une réunion de ses deux parties fermées disjointes $\mathfrak{B}_{1}$ et $\mathfrak{F}_{2} .3^{+}(A)$ est la réunion de ses deux parties fermées

$$
3_{i}=\left\{P \in 3^{+}(A) ; 3^{+}(P) \cap \mathfrak{E}_{i} \neq \phi\right\}, \quad i=1,2 .
$$

Soit $A^{\prime}$ un des points de $3_{1} \cap 3_{2}$ ayant la plus grande abscisse $\alpha^{\prime} . \quad 3^{+}\left(A^{\prime}\right)$ est la réunion de ses deux parties fermées

$$
3_{i}^{\prime}=\left\{P \in 3^{+}\left(A^{\prime}\right) ; 3^{+}(P) \cap \complement_{i} \neq \phi\right\}, \quad i=1,2 .
$$

Puisqu'on a les inclusions $3_{1}^{\prime} \subset \bigotimes_{1}, 3_{2}^{\prime} \subset \bigotimes_{2}$, l'intersection $3_{1}^{\prime} \cap 3_{2}^{\prime}$ ne contient que le point $A^{\prime}$. Donc la section de $3^{+}\left(A^{\prime}\right)$ par l'ayperplan $t=\tau$ ne peut être un cortinu quelque petite que soit la différerce $\tau-\alpha>0$.

Proposision 6.3. Soit F une famille de caractéristiques satisfaisant à l'une cies trois conditions de la proposition 6. 1. Si $\widetilde{F}^{+}(P)$

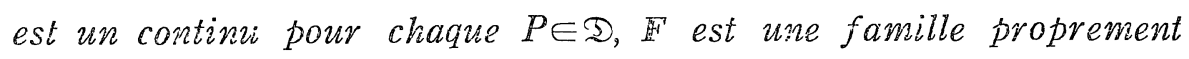
knesérienne à droite, et réciproquement.

En effet, supposons la condition de la proposition remplie. Soit 
$A \in \mathfrak{D}$ un point d'abscisse $\alpha$ qui n'appartient pas à $\mathfrak{B}^{d}$. $\mathfrak{S}^{d}$ étant fermé, la distance $\operatorname{dist}\left(A, \mathfrak{B}^{d}\right)$ est positive. Si donc $\tau-\alpha$ est positive et assez petite, toute caractéristique de $\widetilde{\boldsymbol{F}}^{+}(A)$ perce l'hypeplan $t=\tau$. En faisant correspondre à $\mathfrak{x} \in \widetilde{\boldsymbol{F}}^{+}(A)$ le point de recontre de $\mathfrak{x}$ avec l'hyperplan $t=\tau$, on obtient une application continu de $\widetilde{\boldsymbol{F}}^{+}(A)$ sur la section $\subseteq$ de $3^{+}(A)$ par l'hyperplan $t=\tau$, et puisque $\widetilde{\boldsymbol{F}}^{+}(A)$ est un continu, $\subseteq$ l'est aussi. $A$ est donc un point proprement knesérien à droite.

La réciproque sera démontrée si l'on montre que $\widetilde{F}^{+}(A)$ est bien enchaîné.

Soit $\beta$ la plus grande des abscisses des points de $3^{+}(A)$. Si l'abscisse de $B \in B^{+}(A)$ est $\beta, \mathbb{F}^{+}(B)$ ne contient que $B$.

Supposons que si l'abscisse d'un point $P \in 3^{+}(A)$ est supérieure à $\gamma$, deux éléments quelconques de $\widetilde{F}^{+}(P)$ peuvent se joindre par une chaîne d'éléments de $\widetilde{F}^{+}(P)$ à chaînons inférieurs à $\varepsilon$ et considérens un point $P \in R^{+}(A)$ d'abscisse $r$. Si $P$ est un point extrême droit de $D, \widetilde{F}^{+}(P)$ ne contient que $P$. Sinon toutes les caractéristiques de $\widetilde{F}^{+}(P)$ existent dans un intervalle assez petit $[r, \tau]$, et la section $\subseteq$ de $3^{+}(P)$ par l'hyperplan $t=\tau$ est un continu.

Prenons deux éléments quelconques $x$ et $y$ de $\widetilde{T^{\prime}} \div(P)$. Ils percent l'hyperplan $t=\tau$ en des points $P^{\prime}$ et $Q^{\prime}$. Désignons par $c_{c}^{\prime \prime}$ l'arc de $x$ limité par $P^{\prime}$ et $\mathfrak{B}^{d}$ et par $\mathfrak{y}^{\prime}$ celui de $\mathfrak{y}$ limité par $Q^{\prime}$ et $\mathfrak{S}^{d}$. On peut former, comme dans la démonstration de la proposition 5.2, une chaîne d'éléments de $\widetilde{F}^{+}(\subseteq)$ à chaînons inférieurs à $\varepsilon ; \mathfrak{x}^{\prime}=\mathfrak{x}_{1}^{\prime}, \mathfrak{x}_{2}^{\prime}, \cdots, \mathfrak{x}_{m}^{\prime}=\mathfrak{y}^{\prime}$. En joignant les extrémités gauches de ces caractéristiques au point $P$ par des arcs de caractéristiques, on obtient une chaîne d'éléments de $\widetilde{F}^{+}(P)$ à chaînons inférieurs à $\varepsilon$.

Prenons une valeur $\gamma^{\prime}$ telle que $r-\gamma^{\prime}$ est positive et assez petite. Soit $P$ un point de $3^{+}(A)$ dont l'abscisse est égale à $\gamma^{\prime}$. Si deux caractéristiques $x$ et $\mathfrak{y}$ de $\widetilde{F}^{+}(P)$ percent l'hyperplan $t=r$, on peut construire de la même manière une chaîne d'éléments de $\widetilde{F}^{+}(P)$ à chaînons inférieurs à $\varepsilon$ joignant $\mathfrak{x}$ et $\mathfrak{y}$. Si les extrémités droites de $\mathfrak{x}$ et $\mathfrak{y}$ ont des abscisses au plus égales à $r$, la distance $\operatorname{Dist}(\mathfrak{x}, \mathfrak{y})$ est inférieure à $\varepsilon$. 
Il nous reste à examiner le cas où une seule des extrémités a une abscisse plus grande que $\gamma$. Alors, puisque $\mathfrak{Z}^{+}(P) \cap \mathfrak{B}^{d}$ est un continu, il existe une caractéristique $z \in \widetilde{\boldsymbol{F}}^{+}(P)$ dont l'extrémité droite a une abscisse égale à $\gamma$. On peut joindre $x$ à $z$ et $\hat{\gamma}$ à $\mathfrak{y}$ respectivement par une chaîne d'éléments de $\widetilde{\boldsymbol{F}}^{+}(P)$ à chaînons inférieurs à $\varepsilon$.

D'après ce qui précède, nous pouvons conclure que pour tout $P \in \mathcal{B}^{+}(A)$ deux éléments quelconques de $\widetilde{\boldsymbol{F}}^{+}(P)$ peuvent être reliés par une chaîne d'éléments de $\widetilde{\boldsymbol{F}}^{+}(P)$ à chaînons inférieurs à $\varepsilon$. $\widetilde{\boldsymbol{F}}^{+}(A)$ est donc bien enchaîné.

Les propositions 6.1 et 6.2 entraînent immédiatement la

Proposition 6.4. Soit $\mathbb{F}$ une famille proprement knesérienne à droite telle que $\mathfrak{B}^{d}$ est une partie fermée de $\mathfrak{B} . \mathfrak{Z}^{+}(\mathfrak{F}) \cap \mathfrak{Z}^{d}$ est alors un continu si (5 est une partie continue de $\mathfrak{D}$.

Soit $\mathfrak{s}$ une partie fermée de $\mathfrak{D}$. Si $\mathbb{G}=\mathbb{F}^{+}(\mathfrak{F})$, on a évidemment

$$
\widetilde{\boldsymbol{G}}^{+}(P)=\widetilde{\boldsymbol{F}}^{+}(P)
$$

pour $P \in 3^{+}(\mathbb{S})$. On a donc la

Proposition 6.5. Soit $\boldsymbol{F}$ une famille proprement knesérienne à droite et ‘્s une partie fermée de D. Alors $\mathbb{F}^{+}(\mathfrak{5})$ est une famille proprement knesérienne à droite.

\section{Section transversale.}

Définition 7.1. Une partie compacte $\subseteq$ de $\mathfrak{D}$ est appelée section transversale de $\mathfrak{D}$ si chaque caractéristique maximale rencontre $\mathfrak{S}$ en un point et en un seul.

Il est clair que si $\mathfrak{B}^{d}$ est compacte, elle est une section transversale.

Proposition 7.1. Soit $\subseteq$ une section transversale de $\mathbb{D}$ et $\mathscr{F}$ l'ensemble des caractéristiques qui contiennent des points de 5 . Alors l'application qui fait correspondre à une caractéristique de $\mathscr{F}$ son intersection avec $\subseteq$ est continue.

Considérons en effet une suite de caractéristiques $\left\{\hat{x}_{k}\right\}$ extraite de $\mathscr{I}$ et convergeant vers $\mathfrak{x}$. $\mathfrak{x}$ appartient évidemment à $\mathscr{F}$. On a d'autre part 


$$
\lim \sup \left(\underline{x}_{k} \cap \mathfrak{S}\right) \subset \mathfrak{x} \cap \mathfrak{S},
$$

d'où résulte la continuité de l'application.

Puisqu'une image continue d'un continu compact est aussi un continu compact, on a la

Próposition 7.2. Soit $\mathbb{F}$ une famille proprement knesérienne $\grave{a}$ droite. Si $\mathfrak{5}$ est une partie continue de $\mathfrak{D}$, une section transversale de $3^{+}($(5) est un continu.

Définition 7.2. Soit $\subseteq$ une section transversale de $\mathfrak{D}$. Si un point $A$ de $\mathscr{D}$ n'est pas sur $\mathfrak{S}$, il existe au moins une caractéristique dont l'une des extrémités est $A$ et l'autre $A^{\prime}$ se trouve dans $\mathfrak{S}$. Nous dirons que $A$ est à gauche ou $\grave{a}$ droite de $\subseteq$ suivant que l'abscisse de $A$ est plus petite ou plus grande que celle de $A^{\prime}$.

Remarane. Considérons deux caractéristiques $x_{1}$ et $x_{2}$ dont $A$ est une de leurs extrémités. Les autres extrémités $P_{1}$ et $P_{2}$ qui se trouvent sur $\mathfrak{S}$ sont situées d'un même côté de $A$. Car sinon, on obtiendrait, d'après la propriété $4^{\circ}$, une caracteristique dont les extrémités sont $P_{1}$ et $P_{2}$. C'est impossible puisque $\sqrt{ }$ est une section transversale.

Définition 7. 3. Soit $\mathfrak{S}$ une section transversale de $\mathfrak{D}$. L'ensemble des points de $\mathfrak{D}$ situés à gauche de $\mathfrak{S}$ ou sur $\mathfrak{S}$ est appelé ensemble (D) tronqué à droite par $\mathfrak{S}$ et nous le désignons par $\mathfrak{D}_{\mathfrak{S}}^{d}$. Nous définissons de même ensemble $\mathfrak{D}$ tronqué à gauche par $S$ et nous le désignons par $\mathfrak{S}_{\mathfrak{S}}^{g}$.

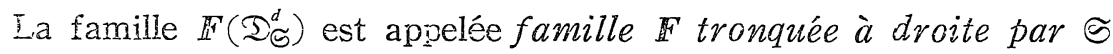
et nous la désignons par $\mathbb{F}_{\circlearrowleft}^{d}$. Nous défnissons de même famille $\mathbb{F}$ tronguée à gauche par $\mathfrak{S}$ et nous la désignonos par $\mathbb{F}_{\subseteq}^{g}$.

IEs domaires fondamentaux des familles $\mathbb{F}_{\subseteq}^{d}$ et $\mathbb{F}_{\Im}^{g}$ sont $\mathfrak{S}_{\subseteq}^{d}$ et $\mathfrak{T}_{\mathscr{S}}^{g}$. Nous désignons les frontières de $\mathfrak{D}_{\mathscr{S}}^{d}$ et $\mathfrak{D}_{\mathscr{S}}^{g}$ par $\mathfrak{R}_{\mathscr{S}}^{d}=\mathfrak{B}\left(\mathbb{F}^{d}\right)$ et $\mathfrak{R}_{\mathscr{S}}^{b}=$

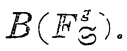

On obtient immédiatement la proposition suivante, qui est une généralisation de la proposition 4. 1 .

Proposition 7. 3. Soit $\mathbb{F}$ une famille knesérienne à droite. Si 
(c) est une section transversale de $\mathfrak{D}$, les familles $\mathbb{F}^{d}$ et $\mathbb{F}_{(5}^{g}$ sont aussi knesériennes à droite.

\section{Un lemme.}

Lemme. Soit $F^{\prime}$ une famille de caractéristiques dont le domaine fondanental est $\mathfrak{D}^{\prime}$ et $\mathfrak{D}$ une partie compacte de $\mathfrak{D}^{\prime}$. Soit $A$ un point de $\mathfrak{B}_{+}(\mathbb{F})$, où $\mathbb{F}=\mathbb{F}^{\prime}(\mathfrak{D})$, et supposons que $A$ soit un point intérieur de $\mathfrak{D}^{\prime}$ ou un point de $\mathfrak{B}^{+}\left(\mathbb{F}^{\prime}\right)$. Nous supposons de plus que les conditions suivantes soient remplies:

$1^{\circ}$ Toute caractéristique de ${\widetilde{\mathbb{F}^{\prime}}}^{\prime}(A)$ existe dans un intervalle assez petit $[\alpha, \alpha+\delta], \alpha$ désignant l'abscisse de $A$;

$2^{\circ} \mathscr{I}=\boldsymbol{F}_{\alpha+\delta}^{\prime+}(A)$ est une famille proprement knesérienne à droite;

$3^{\circ}$ Une caractéristique de $\mathbb{F}_{\alpha+\delta}^{\prime+}(A)$ issue d'un point extérieur de $\stackrel{2}{2}$ ne peut atteindre à la frontière $\mathfrak{B}$ de $\mathfrak{D}$.

$4^{\circ} \quad i_{\alpha+\delta}^{+}(A ; F)$ ne contient aucun point de $\mathfrak{B}^{+}(\mathbb{F})$.

$A$ est alors un point knesérien à droite de la famille $\boldsymbol{F}$.

Supposons en effet que la réunion de $3_{\alpha+\delta}^{+}(A ; \boldsymbol{F}) \cap \mathfrak{B}(\boldsymbol{F})$ et de la section de $3^{+}(A ; \boldsymbol{F})$ par l'hyperplan $t=\alpha+\delta$ fût une réunion de ses deux parties fermées disjointes $\mathfrak{F}_{1}$ et $\mathfrak{F}_{2}$.

Désignons par $\mathscr{E}$ l'ensemble des caractéristiques $\mathfrak{x}$ de $\widetilde{\boldsymbol{F}}_{\alpha+\delta}^{\prime+}(A)$ telles que si l'on prend convenablement une valeur $\beta \in[\alpha, \alpha+\delta]$, on ait $(\beta, \mathfrak{x}(\beta)) \in \mathcal{F}_{1}$ et $(t, \mathfrak{x}(t)) \notin \mathfrak{D}$ dans l'intervale $(\beta, \alpha+\delta]$. $\mathscr{F}$ est une partie fermée de $\mathscr{F}^{\prime}={\widetilde{F_{\alpha+\delta}^{\prime}}}^{+}(A)$.

En effet, considérons une suite $\left\{\Upsilon_{k}\right\}$ extraite de $\mathscr{H}$ convergeant vers $\mathfrak{x}$. Il existe une valeur $\beta_{k} \in[\alpha, \alpha+\delta]$ telle que l'on ait $\left(\beta_{k}, \mathfrak{x}\left(\beta_{k}\right)\right) \in \mathfrak{S}_{1}$ et $(t, \mathfrak{x}(t)] \oplus D$ pour $\beta_{k}<t \leqq \alpha+\delta$. En prenant une suite partielle, s'il est nécessaire, on peut supposer $\beta_{k} \rightarrow \beta^{\prime}$. Le point $\left(\beta^{\prime}, \mathfrak{x}\left(\beta^{\prime}\right)\right)$ appartient à $\mathfrak{F}_{1}$. Soit $\beta$ la valeur telle que l'on ait $(\beta, \mathfrak{x}(\beta)) \in \mathfrak{B}(\boldsymbol{F})$ et $(t, \mathfrak{x}(t)) \notin \mathfrak{A}$ pour $\beta<t \leqq \alpha+\delta$. On a alors $\beta^{\prime} \leqq \beta$ et d'après l'hypothèse $3^{\circ}$ l'arc de $\mathfrak{x}$. dont les extrémités sont $(\beta, \mathfrak{C}(\beta))$ et $\left(\beta^{\prime}, \mathfrak{x}\left(\beta^{\prime}\right)\right)$ est contenu dans $\mathfrak{B}(\boldsymbol{F})$, de sorte qu'il est contenu dans $\mathfrak{F}_{1} \cup \mathfrak{F}_{2}$. $\mathfrak{F}_{1}$ et $\mathfrak{F}_{2}$ étant des 
ensembles fermés disjoints, il est contenu dans $\mathfrak{F}_{1}$ et la caractéristique $\mathfrak{x}$ appartient à $\mathscr{F}$.

La même considération nous montre que $\mathscr{F}^{\prime}-\mathscr{F}$ est aussi une partie fermée de $\mathscr{F}^{\prime}$. $\mathscr{F}^{\prime}$ étant une famille proprement knesérienne à droite, $\mathscr{F}^{\prime}$ est un continu dans $\operatorname{Comp}\left(R \times R^{n}\right)$, car la frontière droite de $\gtrless_{\alpha+\delta}^{+}(A)$ est la section de $3^{+}(A)$ par l'hyperplan $t=\alpha+\delta$. Donc l'intersection $\mathscr{F} \cap\left(\mathscr{F}^{\prime}-\mathscr{F}\right)$ ne serait pas vide. C'est évidemment impossible.

Par suite l'un des ensembles $\mathscr{F}$ et $\mathscr{F}^{\prime}-\mathscr{F}$ est vide. Supposons par exemple $\mathscr{F}=\phi$. Alors $\mathfrak{F}_{1}$ ne peut contenir de points d'abscisse $\alpha+\delta$ et la plus grande des abscisses de ses points a une valeur $\beta<\alpha+\delta$. Les points de $\mathfrak{S}_{1}$ ayant l'abscisse égale à $\beta$ ne peuvent appartenir à $\mathfrak{B}_{+}(\boldsymbol{F})$. D'après l'hypothèse $4^{\circ}$, ils appartiennent à $\mathfrak{B}^{d}(\boldsymbol{F})$ et d'après l'hypothèse $3^{\circ}$ les caractéristiques de $\boldsymbol{F}^{\prime+}(A)$ passant par eux appartiennent à $\mathscr{F}$.

Or, c'est impossible, parce que nous supposions $\mathscr{F}=\phi$. Le lemme est donc établi.

\section{Problème aux limites.}

Soit $\boldsymbol{F}$ une famille knesérienne à droite. Le problème aux limites s'énonce alors comme il suit:

Trouver une caractéristique dont les extrémités se trouvent dans des parties continues $\mathfrak{F}^{\mathrm{S}}$ et $\mathfrak{F}^{\prime}$ données respectivement dans $\mathfrak{B}^{\mathfrak{g}} \cup \mathfrak{B}_{\text {_ et }} \mathfrak{B}^{d} \cup \mathfrak{B}_{+}$.

D'après la proposition 4.2 , l'ensemble $\mathfrak{S}=\mathfrak{3}^{+}(\mathfrak{F}) \cap\left(\mathfrak{B}^{d} \cup \mathfrak{B}_{+}\right)$est un continu. Si donc l'intersection $\mathfrak{S}=\left(\mathfrak{S} \cap\left(\mathfrak{B}^{d} \cup \mathfrak{B}_{+}\right)\right.$se décompose en deux parties fermées $\mathfrak{F}_{1}$ et $\mathfrak{F}_{2}$ séparées par $\mathfrak{G}^{\prime}$ dans $\mathfrak{B}^{d} \cup \mathfrak{B}_{+}$, $\mathfrak{S}$ contient des points de $\mathfrak{r}^{\prime}$. Si $Q$ est un point de l'intersection $\mathfrak{S} \cap \mathfrak{G}^{\prime}, \mathbb{Z}^{+}(\mathfrak{r})$ contient une caractéristique dont $Q$ est l'extrémité droite. Nous obtenons donc la

Proposition 9.1. Soit $\mathbb{F}$ une famille knesérienne à droite et soient $\mathfrak{G}_{\text {s }}$ et $\mathfrak{G}^{\prime}$ des continus donnés respectivement dans $\mathfrak{B}^{g} \cup \mathfrak{B}_{\text {_ et }}$ $\mathfrak{B}^{d} \cup \mathfrak{B}_{+}$. Si l'intersection $\mathfrak{F}_{\mathfrak{S}} \cap\left(\mathfrak{B}^{d} \cup \mathfrak{B}_{+}\right)$se décompose en deux parties fermées séparées par $\mathfrak{G}^{\prime}$ dans $\mathfrak{B}^{d} \cup \mathfrak{B}_{+}$, il existe au moins une caractéristique dont les extrémités se trouvent respectivement dans $\mathfrak{F}$ et $\mathfrak{G}^{\prime}$. 


\section{Théorème d'existence de M. Nagumo et celui de $H$. Okamura.}

Considérons l'équation différentielle du second ordre

$$
x^{\prime \prime}=f\left(t, x, x^{\prime}\right) \text {. }
$$

La fonction $f$ est supposée continue dans le domaine fermé $\mathfrak{D}$ :

$$
0 \leqq t \leqq 1, \underline{\omega}(t) \leqq x \leqq \bar{\omega}(t), \underline{\Omega}(t, x) \leqq y \leqq \bar{\Omega}(t, x)
$$

où $\underline{\omega}$ et $\bar{\omega}$ sont des fonctions deux fois continûment dérivables dans l'intervalle $[0,1]$ et $\underline{\Omega}$ et $\bar{\Omega}$ sont des fonctions admettant des dérivées continues dans le domaine fermé

$$
0 \leqq t \leqq 1, \quad \underline{\omega}(t) \leqq x \leqq \bar{\omega}(t) .
$$

Supposons de plus que l'on ait les inégalités

$$
\begin{gathered}
\underline{\Omega}(t, \underline{\omega}(t)) \leqq \omega^{\prime}(t) \leqq \bar{\Omega}(t, \underline{\omega}(t)), \\
\underline{\Omega}(t, \bar{\omega}(t)) \leqq \bar{\omega}^{\prime}(t) \leqq \bar{\Omega}(t, \bar{\omega}(t)) ; \\
\quad \underline{\omega}^{\prime \prime}(t) \geqq f\left(t, \underline{\omega}(t), \underline{\omega}^{\prime}(t)\right), \\
\bar{\omega}^{\prime \prime}(t) \geqq f\left(t, \bar{\omega}(t), \bar{\omega}^{\prime}(t)\right)
\end{gathered}
$$

dans l'intervalle $[0,1]$ et les inégalités

$$
\begin{aligned}
& f(t, x, \underline{\Omega}(t, x))-\underline{\underline{\Omega}_{t}}(t, x)-\underline{\Omega_{x}}(t, x) \underline{\underline{\Omega}}(t, x)>0, \\
& f(t, x, \bar{\Omega}(t, x))-\bar{\Omega}_{t}(t, x)-\overline{\bar{\Omega}_{x}}(t, x) \bar{\Omega}(t, x)<0
\end{aligned}
$$

dans le domaine (10.3).

Théorème d'existence de M. Nagumo s'énonce alors:

Sous ces hypothèses, si l'on a

$$
\underline{\omega}(0)=b=\bar{\omega}(0), \quad \underline{\omega}(1) \leqq b^{\prime} \leqq \bar{\omega}(1),
$$

il existe au moins une solution telle que l'on ait

$$
y(0)=b, \quad y(1)=b^{\prime}
$$

H. Okamura suppose les mêmes hypothèses relatives à $\underline{\omega}$ et $\bar{\omega}$. La fonction $f(t, x, y)$ est supposée continue dans le domaine non borné

$$
0 \leqq t \leqq 1, \quad \underline{\omega}(t) \leqq x \leqq \bar{\omega}(t), \quad-\infty<y<+\infty .
$$


Soit $\psi$ une fonction lipschitzienne telle que $\underline{\omega} \leqq \psi \leqq \bar{\omega}$ dans lintervalle $[0,1]$. Il suppose l'existence des forictions continues $\Phi_{1}, \Phi_{2}, \Psi_{1}, \Psi_{2}$, qui sont positives respectivement dans les domaines

$$
\begin{aligned}
& 0 \leqq t \leqq 1, \quad \psi(t) \leqq x \leqq \bar{\omega}(t), \quad y \geqq K ; \\
& 0 \leqq t \leqq 1, \quad \omega(t) \leqq x \leqq \psi(t), \quad y \leqq-K \text {; } \\
& 0 \leqq t \leqq 1, \quad \underline{\omega}(t) \leqq x \leqq \psi(t), \quad y \geqq K ; \\
& 0 \leqq t \leqq 1, \quad \psi(t) \leqq x \leqq \bar{\omega}(t), \quad y \leqq-K
\end{aligned}
$$

et admettent les dérivés continues dans leurs intérieurs, où $\mathbb{K}$ est une certaine constante positive. Elles convergent uniformément vers 0 lorsque $|y| \rightarrow \infty$. Il suppose de plus les inégalités suivantes remplies:

$$
\begin{aligned}
& \frac{\partial \Phi_{i}}{\partial t}+\frac{\partial \Phi_{i}}{\partial x} y+\frac{\partial \Phi_{i}}{\partial y} f(t, x, y) \geqq 0, \quad i=1,2, \\
& \frac{\partial \Psi_{i}}{\partial t}+\frac{\partial \Psi_{i}}{\partial x} y+\frac{\partial \Psi_{i}}{\partial y} f(t, x, y) \leqq 0, \quad i=1,2 .
\end{aligned}
$$

Théorème d'existence de $\mathrm{H}$. Okamura s'énonce alors:

Il existe au moins une solution telle que l'on ait

$$
y(0)=\psi(0), \quad y(1)=\psi(1) .
$$

\section{Examen de la condition d'existence de M. Nagumo.}

Désignons par $\mathbb{F}$ l'ensemble des solutions du système différentiel

$$
x^{\prime}=y, \quad y^{\prime}=f(t, x, y),
$$

qui est équivalent à l'équation (10.1).

On sait que tout point intérieur du domaine $\mathscr{D}$ est knesérien. On voit aussi sans peine que tout point de $\mathfrak{B}^{g} \cup \mathfrak{B}^{-}$est knesérien à gauche.

Les points de $\mathfrak{B}$ qui se trouvent sur l'hyperplan $t=0$ appartiennent à $\mathfrak{S}^{g}$.

Le point $(\tau, \xi, \eta)$ tel que l'on ait

$$
\tau=1, \quad \underline{\omega}(1)<\xi<\bar{\omega}(1), \quad \underline{\Omega}(1, \xi)<\eta<\bar{\Omega}(1, \xi),
$$

appartient à $\mathfrak{B}^{-}$.

Le point $(\tau, \xi, \eta)$ tel que l'on ait 


$$
0<\tau \leqq 1, \quad \underline{\omega}(\tau) \leqq \xi \leqq \bar{\omega}(\tau), \quad \eta=\bar{\Omega}(\tau, \xi)
$$

ou

$$
0<\tau \leqq 1, \quad \underline{\omega}(\tau) \leqq \xi \leqq \bar{\omega}(\tau), \quad \eta=\underline{\Omega}(\tau, \xi)
$$

appartient à $\mathfrak{B}^{g}$. C'est ce que l'on voit sans peine à l'aide des inégalités (10.6).

Le point $(\tau, \xi, \eta)$ tel que l'on ait

$$
0<\tau \leqq 1, \quad \xi=\underline{\omega}(\tau), \quad \underline{\omega^{\prime}}(\tau)<\eta<\bar{\Omega}(\tau, \xi)
$$

ou

$$
0<\tau \leqq 1, \quad \xi=\bar{\omega}(\tau), \quad \underline{\Omega}(\tau, \xi)<\eta<\bar{\omega}^{\prime}(\tau)
$$

appartient à $\mathfrak{B}^{g}$ et le point $(\tau, \xi, \eta)$ tel que l'on ait

$$
0<\tau \leqq 1, \quad \xi=\underline{\omega}(\tau), \quad \underline{\Omega}(\tau, \xi)<\eta<\underline{\omega}^{\prime}(\tau)
$$

ou

$$
0<\tau \leqq 1, \quad \xi=\bar{\omega}(\tau), \quad \bar{\omega}^{\prime}(\tau)<\eta<\bar{\Omega}(\tau, \xi)
$$

appartient à $\mathfrak{B}^{-}$. On obtient ces conclusions en comparant $\eta$ avec $\underline{\omega}^{\prime}(\tau)$, $\bar{\omega}^{\prime}(\tau)$.

Nous allons montrer à l'aide du lemme que le point $A(\tau, \xi, \eta)$ tel que l'on ait

$$
0<\tau \leqq 1, \quad \xi=\bar{\omega}(\tau), \quad \eta=\bar{\omega}^{\prime}(\tau)
$$

est knesérien à gauche.
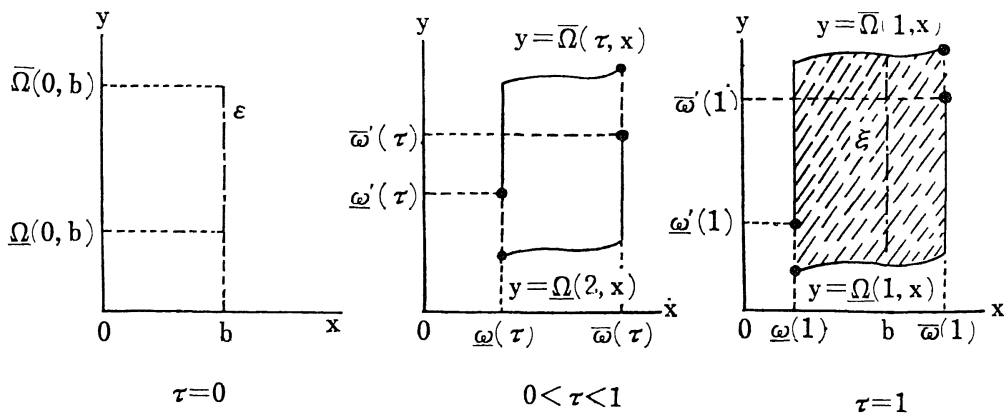

$B^{g} \cup B_{-}$ 
Supposons l'existence d'une solution $x=\varphi(t), y=\varphi^{\prime}(t)$ prenant les valeurs $\xi, \eta$ pour $t=\tau$. Si la solution existe dans un intervalle $\left[\tau^{\prime}, \tau\right]$, on a $\varphi(t) \leqq \bar{\omega}(t)$ dans l'intervalle et

$$
\varphi(\tau)=\bar{\omega}(\tau), \quad \varphi^{\prime}(\tau)=\bar{\omega}^{\prime}(\tau),
$$

ce qui implique l'existence d'une suite de valeurs $\left\{\tau_{k}\right\}$ telle que l'on ait

$$
\tau_{k} \uparrow \tau, \quad \varphi^{\prime}\left(\tau_{k}\right) \geqq \bar{\omega}^{\prime}\left(\tau_{k}\right) .
$$

On en déduit

ou

$$
\begin{aligned}
& \varphi^{\prime \prime}(\tau) \leqq \bar{\omega}^{\prime \prime}(\tau) \\
& \bar{\omega}^{\prime \prime}(\tau) \geqq f\left(\tau, \varphi(\tau), \varphi^{\prime}(\tau)\right) .
\end{aligned}
$$

Si donc on a l'inégalité stricte

$$
\bar{\omega}^{\prime \prime}(\tau)<f\left(\tau, \bar{\omega}(\tau), \bar{\omega}^{\prime}(\tau)\right),
$$

le point $(\tau, \xi, \eta)$ appartient à $\mathfrak{B}^{g}$.

Il suffit donc de considérer le cas où l'on a l'égalité

$$
\bar{\omega}^{\prime \prime}(\tau)=f\left(\tau, \bar{\omega}(\tau), \bar{\omega}^{\prime}(\tau)\right) .
$$

Si l'on avait

$$
\bar{\omega}^{\prime}(\tau)=\bar{\Omega}(\tau, \bar{\omega}(\tau)),
$$

la dérivée de $\bar{\omega}^{\prime}(t)-\bar{\Omega}(t, \bar{\omega}(t))$ aurait la valeur négative

$$
\begin{aligned}
& \bar{\omega}^{\prime \prime}(\tau)-\bar{\Omega}_{t}(\tau, \bar{\omega}(\tau))-\bar{\Omega}_{x}(\tau, \bar{\omega}(\tau)) \bar{\omega}^{\prime}(\tau) \\
= & f\left(\tau, \bar{\omega}(\tau), \bar{\omega}^{\prime}(\tau)\right)-\bar{\Omega}_{t}(\tau, \bar{\omega}(\tau))-\bar{\Omega}_{x}(\tau, \bar{\omega}(\tau)) \bar{\Omega}(\tau, \bar{\omega}(\tau))
\end{aligned}
$$

pour $t=\tau$, d'où résulterait l'inégalité

$$
\bar{\omega}^{\prime}(t)>\bar{\Omega}(t, \bar{\omega}(t))
$$

dans un intervalle assez petit $\left[\tau^{\prime}, \tau\right]$ contrairement à notre hypothèse.

On a donc nécessairement

$$
\bar{\omega}^{\prime}(t)<\bar{\Omega}(t, \bar{\omega}(t))
$$

pour $0<t \leqq 1$. On a de même

$$
\bar{\omega}^{\prime}(t)>\underline{\Omega}(t, \bar{\omega}(t)) .
$$

Posons 


$$
\underline{\Omega}(t, x)=\underline{\Omega}(t, \bar{\omega}(t)), \quad \bar{\Omega}(t, x)=\bar{\Omega}(t, \bar{\omega}(t))
$$

pour

$$
0 \leqq t \leqq 1, \quad \bar{\omega}(t)<x<\infty
$$

et

$$
f(t, x, y)=f(t, \bar{\omega}(t), y)+r[x-\bar{\omega}(t)]
$$

pour

$$
0 \leqq t \leqq 1, \quad \bar{\omega}(t)<x<\infty, \quad \underline{\Omega}(t, x) \leqq y \leqq \bar{\Omega}(t, x),
$$

où $r$ est une constante positive.

Nous voulons appliquer le lemme, en intervertissant droite et gauche. Dans notre présent cas, la famille $\boldsymbol{F}^{\prime}$ sera l'ensemble des solutions du système différentiel (11.1) dont le domaine fondamental $\mathfrak{D}^{\prime}$ est

$$
0 \leqq t \leqq 1, \quad \underline{\omega}(t) \leqq x \leqq \bar{\omega}(t)+\lambda, \quad \underline{\Omega}(t, x) \leqq y \leqq \bar{\Omega}(t, x),
$$

où $\lambda$ est une constante positive quelconque.

Si $\delta$ est un nombre positif assez petit, $\beta_{\alpha-\delta}^{-}\left(A ; \boldsymbol{F}^{\prime}\right)$ se trouve à l'intérieur de $\mathfrak{D}^{\prime}$ et les deux premières conditions $1^{\circ}$ et $2^{\circ}$ sont évidemment remplies.

Considérons le domaine

$$
0 \leqq \tau-t \leqq \delta . \quad \varepsilon \leqq x-\bar{\omega}(t) \leqq \lambda, \quad \bar{\omega}^{\prime}(t) \leqq y \leqq \bar{\Omega}(t, \bar{\omega}(t))
$$
où $\varepsilon$ est un nombre positif. Si $y=\bar{\omega}^{\prime}(t)$,

$$
f(t, x, y) \geqq f\left(t, \bar{\omega}(t), \bar{\omega}^{\prime}(t)\right)+\gamma \varepsilon>\bar{\omega}^{\prime \prime}(t) .
$$

$\varepsilon$ pouvant être supposer aussi petit que l'on veut, on peut en conclure que si l'on marche vers gauche le long d'une caractéristique, on ne peut pénétrer dans le domaine

$$
0 \leqq \tau-t \leqq \delta, \quad 0 \leqq x-\bar{\omega}(t) \leqq \lambda, \quad \bar{\omega}^{\prime}(t) \leqq y \leqq \bar{\Omega}(t, \bar{\omega}(t)),
$$

en franchissant la frontière où l'on a $y=\bar{\omega}^{\prime}(t)$.

Si $x=\bar{\omega}(t)+\varepsilon$, l'inégalité $y>\bar{\omega}^{\prime}(t)$ montre que l'on ne peut pénétrer non plus dans le domaine (11.11) en franchissant la frontière où l'on a $x=\bar{\omega}(t)+\varepsilon$.

Par conséquent, $Z_{\tau+\delta}^{+}\left(A, \boldsymbol{F}^{\prime}\right)$ ne contient aucun point du domaine 
(11. 12) sauf les points $(t, x, y)$ où l'on a $x=\bar{\omega}(t), y=\bar{\omega}^{\prime}(t)$.

Considérons maintenant le domaine

$$
0 \leqq \tau-t \leqq \delta, \quad \varepsilon \leqq x-\bar{\omega}(t) \leqq \lambda, \quad \underline{\Omega}(t, \bar{\omega}(t)) \leqq y \leqq \bar{\omega}^{\prime}(t) .
$$

Comme nous avons vu, on a l'inégalité

$$
f(t, x, y)>\bar{\omega}^{\prime \prime}(t),
$$

pour $y=\bar{\omega}^{\prime}(t)$. Si $x=\bar{\omega}(t)+\varepsilon$, on a l'inégalité $y<\bar{\omega}^{\prime}(t)$ sauf au point $\left(t, \bar{\omega}(t)+\varepsilon, \bar{\omega}^{\prime}(t)\right)$. Par suite si l'on marche vers gauche le long d'une caractéristique issue d'un point du domaine (11.13), on ne peut en sortir, en franchissant la frontière où l'on a $y=\bar{\omega}^{\prime}(t)$ ou $x=\bar{\omega}(t)+\varepsilon$.

D'après ces considérations nous pouvons conclure que la condition $3^{\circ}$ du lemme est remplie.

$\mathcal{Z}_{\alpha-\delta}(A ; \boldsymbol{F})$ ne contient aucun des points frontières de $\mathfrak{D}$ tels que l'on ait

$$
0<\tau-t \leqq \delta, \quad x=\bar{\omega}(t), \quad \bar{\omega}^{\prime}(t)<y \leqq \bar{\Omega}(t, y),
$$

car ils appartiennent à $\mathfrak{B}^{d}(\boldsymbol{F})$. D'après ce que nous avons déjà remarqué, si l'on a

$$
0<\tau-t \leqq \delta, \quad x=\bar{\omega}(t), \quad \underline{\Omega}(t, y)<y<\bar{\omega}^{\prime}(t),
$$

le point $(t, x, y)$ appartient à $\mathfrak{B}^{g}$.

La condition $4^{\circ}$ du lemme sera donc vérifiée, si l'on montre que le point où l'on a $x=\bar{\omega}(t), y=\bar{\omega}^{\prime}(t)$ appartient à $\mathfrak{B}^{g}(\boldsymbol{F}) \cup \mathfrak{B}_{-}(\boldsymbol{F})$. Il suffit pour cela de montrer que si $A \notin \mathfrak{B}^{g}(\boldsymbol{F}), A$ appartient à $\mathfrak{B}_{-}(\boldsymbol{F})$.

L'hypothèse $A \notin \mathfrak{B}^{g}(\boldsymbol{F})$ implique que si $\delta$ est assez petit, $\boldsymbol{F}_{\tau-\delta}^{-}(A)$ contient au moins une caractéristique $\mathfrak{x}$ définie dans l'intervalle $[\tau-\delta, \tau]$.

Grâce aux inégalités qui sont vérifiées aux points frontières du domaine (11.13) situés assez voisins de $A$, le domaine

$$
0 \leqq \tau-t \leqq \delta, \quad 0 \leqq x-\bar{\omega}(t) \leqq \lambda, \quad \underline{\Omega}(t, \bar{\omega}(t)) \leqq y \leqq \bar{\omega}^{\prime}(t),
$$

contient une caractéristique $\mathfrak{y}$ issue de $A$ et définie dans l'intervalle $[\tau-\delta, \tau]$. La section de $\mathcal{Z}^{+}\left(A ; \boldsymbol{F}^{\prime}\right)$ par un hyperplan $t=\tau^{\prime}$ est un continu si $\tau-\delta \leqq \tau^{\prime} \leqq \tau$. Puisqu'elle contient les point $\left(\tau^{\prime}, \mathfrak{x}\left(\tau^{\prime}\right)\right)$ et $\left(\tau^{\prime}, \mathfrak{l}\left(\tau^{\prime}\right)\right)$, elle contient un point $P$ de $\mathfrak{B}(\mathfrak{D})$. D'après la condition $3^{\circ}$ que 
nous avons vérifiée ci-dessus, une caractéristique de $\boldsymbol{F}^{\prime-}(A) \cap \boldsymbol{F}^{\prime+}(P)$ appartient à $\boldsymbol{F}^{-}(A)$. On en conclut que $A$ appartient à $\mathfrak{B}_{-}(A ; \boldsymbol{F})$.

Appliquons maintenant la proposition 9.1, en intervertissant droite et gauche. On prend pour $\&$ le segment

$$
t=0, \quad x=b, \quad \underline{\Omega}(0, b) \leqq y \leqq \bar{\Omega}(0, b)
$$

et pour $\mathfrak{F}^{\prime}$ le segment

$$
t=1, \quad x=b^{\prime}, \quad \underline{\Omega}\left(1, b^{\prime}\right) \leqq y \leqq \bar{\Omega}\left(1, b^{\prime}\right) .
$$

(F et $\mathfrak{F}^{\prime}$ sont contenus respectivement dans $\mathfrak{B}^{g}$ et $\mathfrak{B}^{d}$. Les extrémités $\left(1, b^{\prime}, \underline{\Omega}\left(1, b^{\prime}\right)\right)$ et $\left(0, b^{\prime}, \bar{\Omega}\left(1, b^{\prime}\right)\right)$ du segment $\left(F^{\prime}\right.$ se trouvent dans $\mathfrak{B}^{g}$. Ils sont séparés par $\mathfrak{F}$ dans $\mathfrak{B}^{g} \cup \mathfrak{B}_{-}$.

La proposition 9.1 est donc applicable et l'existence de solutions satisfaisant à la condition aux limites (10.8) est établie.

\section{Examen de la condition d'existence de H. Okamura.}

On prend pour $\mathfrak{D}$ le domaine tel que la section de $\mathfrak{D}$ par un hyperplan $t=\tau$ soit limitée par les quatre courbes

$$
\begin{array}{ll}
\psi(\tau) \leqq x \leqq \bar{\omega}(\tau), & \Phi_{1}(\tau, x, y)=\varepsilon, \\
\underline{\omega}(\tau) \leqq x \leqq \psi(\tau), & \Psi_{1}(\tau, x, y)=\varepsilon^{\prime}, \\
\underline{\omega}(\tau) \leqq x \leqq \psi(\tau), & \Phi_{2}(\tau, x, y)=\varepsilon, \\
\psi(\tau) \leqq x \leqq \bar{\omega}(\tau), & \Psi_{2}(\tau, x, y)=\varepsilon^{\prime},
\end{array}
$$

et les segments qui les joignent bout à bout comme le montre la figure ci-dessous; $\varepsilon$ et $\varepsilon^{\prime}$ sont des nombres assez petits ainsi que le rapport $\varepsilon / \varepsilon^{\prime}$.

Il est clair que:

$1^{\circ}$ les points de $\mathfrak{D}$ sur l'hyperplan $t=0$ appartiennent à $\mathfrak{B}^{g}$;

$2^{\circ}$ les points de $\mathfrak{D}$ sur l'hyperplan $t=1$ appartiennent à $\mathfrak{B}^{d}$;

$3^{\circ}$ les points intérieurs de la section de $\mathfrak{D}$ par l'hyperplan $t=0$ appartiennent à $\mathfrak{B}^{+}$;

$4^{\circ}$ les points intérieurs de la section de $\mathfrak{D}$ par l'hyperplan $t=1$ appartiennent à $\mathfrak{B}^{-}$. 


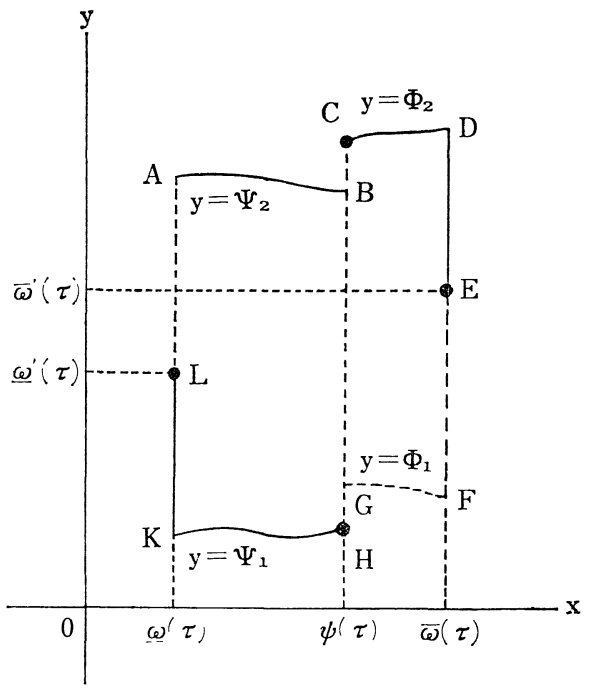

Considérons un point frontière $(\tau, \xi, \eta)$ de la section de $\mathscr{D}$ par l'hyperplan $t=\tau, \tau$ désignant une valeur quelconque telle que $0 \leqq \tau<1$. En raisonnant comme au $\mathrm{n}^{\circ}$ précédent, on voit que:

$5^{\circ}$ les points de la ligne LABC et ceux de la ligne EFGH (les extrémités $\mathrm{L}, \mathrm{C}, \mathrm{E}, \mathrm{H}$ exclues) appartiennent à $\mathfrak{B}^{+}$;

$6^{\circ}$ les points de la ligne CDE et ceux de la ligne HKL (les extrémités $\mathrm{E}, \mathrm{L}$ exclues) appartiennent à $\mathfrak{B}^{d}$;

$7^{\circ}$ les points $\mathrm{E}$ et $\mathrm{L}$ appartiennent à $\mathfrak{S B}^{d} \cup \mathfrak{B}_{+}$.

D'après ces considérations, on voit que la famille $F$ des solutions est knesérienne.

Pour appliquer la proposition 9.1 nous prenons pour $\mathfrak{F}$ et $\xi^{\prime}$ les segment $\mathrm{HC}$ située respectivement dans les hyperplans $t=0$ et $t=1$. Ils sont situés respectivement dans $\mathfrak{B}^{g}$ et $\mathfrak{B}^{d}$. Les extrémités $\mathrm{H}$ et $\mathrm{C}$ de $\mathfrak{F}$ appartiennent à $\mathfrak{B}^{d}$. Elles sont séparées par $\mathfrak{G}^{\prime}$ dans $\mathfrak{B}^{d} \cup \mathfrak{B}_{+}$.

Donc la proposition 9.1 entraîne le théorème d'existence de $H$. Okamura. 


\section{BIBLIOGRAPHIE}

M. Hukuhara,

[1] Sur les systèmes des équations différentielles ordinaires, Proc. Imp. Acad. 4 (1928), 448-449.

[2] Sur l'ensemble des courbes intégrales d'un système d'équations différentielles ordinaires, I, II, III, Ibid. 6 (1930), 360-362; 7 (1931), 37-39; 298-299.

[3] Zyóbibunhóteisiki-ron no Kihonteiri, II, III, Sûbutu Kwaisi, 6 (1932), 134-147; 285-295.

[4] Sur les familles de fonctions à une variable réelle, J. Fac. Sci. Hokkaido Imp. Univ. 1 (1932), 163-209.

[5] Sur les propriétés de la famille des courbes intégrales d'un système différentiel ordinaire. Proc. Japan Acad. 25 (1949), 151-153.

[6] Le problème aux limites pour un système de deux équations différentielles ordinaires, J. Math. Soc. Japan, 3 (1951), 99-103.

[7] Sur un théorème de Kneser, J. Fac. Sci. Univ. Tokyo, 6 (1953), 329-344.

[8] Sur une généralisation d'un théorème de Kneser, Proc. Japan Acad. 29 (1953), 154-155.

[9] Sur l'application qui fait correspondre à un point un continu bicompact, Ibid. 31 (1955), 5-7.

[10] La propriété de Kneser globale et le problème aux limites, Publ. RIMS, Kyoto Univ. Ser. A, 1 (1966), 129-148.

[11] Zyôbibunhôteisikiron (Théorie des équations différentielles ordinaires), Iwanami, Tokyo, 1933.

M. Hukuhara et M. Nagumo,

[1] Un théorème relatif à l'ensemble des courbes intégrales d'un système d'équations différentielles, Proc. Phys.-Math. Soc. Japan, 12 (1930), 233-239.

A. Marchaud,

[1] Sur les champs de demi-cônes et équations différentielles du premier ordre, Bull. Soc. Math. France, 62 (1932), 1-38.

[2] Sur les champs continus de demi-cônes convexes et leurs intégrales, C. R. Acad. Sci. Paris, 199 (1934), 1278-1280.

[3] Sur les champs continus de demi-cônes convexes et leurs intégrales, Comp. Math. 3 (1936), 89-127.

M. Nagumo,

[1] Über das System der gewöhnlichen Differentialgleichungen, Japan. J. Math. 4 (1927), 215-230.

[2] Über die Differentialgleichung $y^{\prime \prime}=f\left(x, y, y^{\prime}\right)$, Proc. Phys.-Math. Soc. Japan, 19 (1937). 861-866.

[3] Kansû Kûkan ni okeru Syazô, Kansû Hôteisiki, No. 4 (1938), 10-22.

[4] Dai-2-kai Zyôbibunhôteisiki no Kyôkaiti Mondai, I, II, Ibid., No. 5 (1939), 27-34; No. 6 (1939), 37-44.

[5] $y^{\prime \prime}=f\left(x, y, y^{\prime}\right)$ no Kyôkaiti Mondai ni tuite, I, II, Ibid., No. 30 (1941), 36-46; No. 17 (1942), 50-52.

[6] Über die Lage der Integralkurven gewöhnlicher Differentialgleichungen, Proc. Phys.-Math. Soc. Japan, 24 (1942), 551-559.

[7] Über das Randwertproblem der nicht linearen gewöhnlichen Differentialgleichungen zweiter Ordnung, Ibid. 24 (1942), 845-851. 
[8] Eine Art der Randwertaufgabe von Systemen gewöhnlicher Differentialgleichungen, I, II, Ibid. 25 (1943), 221-226, 384-390.

[9] Syazôdo to Sonzai Teiri (Degree of mapping and existence theorem), Kawade, Tokyo, 1948.

H. Okamura,

[1] $y^{\prime \prime}=f\left(x, y, y^{\prime}\right)$ ni tuite, I, II, III, Kansû Hôteisiki, No. 27 (1941), 27-35: No. 30 (1941), 14-19: No. 31 (1942), 32-40.

T. Waźewski,

$[1]$ On an optimal control problem (in connection with the theory of orientor fields of A. Marchaud and S. C. Zaremba), Differential Equations and Their Applications: Proceedings of the Conference held in Prague in September 1962 (1963), 229-242.

S. C. Zaremba,

[1] Sur une extension de la notion d'équation différentielle, C. R. Acad. Sci. Paris, 199 (1934), 545-548.

[2] Sur les équations en paratingent, Bull. Sci. Math. 60 (1936), 139-160.

\section{ERRATA}

Au lieu de Lire

Figure à la page 263: $y=\underline{\Omega}(2, x) \rightarrow y=\underline{\Omega}(\tau, x)$

Figure à la page 268: $y=\Phi_{1} \quad \rightarrow \quad y=\Psi_{2}$

$y=\Phi_{2} \quad \rightarrow \quad y=\Phi_{1}$

$y=\Psi_{1} \quad \rightarrow \quad y=\Phi_{2}$

$y=\Psi_{2} \quad \rightarrow \quad y=\Psi_{1}$ 